\title{
Subclinical systemic lymphedema and its progression
}

\section{Jose Maria Pereira de Godoy ${ }^{1}$, Henrique Jose Pereira de Godoy², Ana Carolina Pereira de Godoy ${ }^{3}$, Maria de Fátima Guerreiro Godoy ${ }^{4}$}

${ }^{1}$ Cardiology and Cardiovascular Surgery Department of the Medicine School in São José do Rio Preto (FAMERP), CNPq (National Council for Research and Development), São José do Rio Preto, Brazil, ${ }^{2}$ Student of Medicine School of Universidade Federal do Mato Grosso-Cuiabá-UFMT and Research Group in the Clínica Godoy, Sao Jose do Rio Preto, Brazil, ${ }^{3}$ Resident of the Pediatrics Unit Intensive Therapy of Santa Casa de São Paulo, Brazil and Research Group of Clinica Godoy, São Jose do Rio Preto, Brazil, ${ }^{4}$ Occupational Therapist professor of the Post-Graduate Strictu Sensu in Medicine School in São José do Rio Preto (FAMERP) and Research Group in the Clínica Godoy, Sao Jose do Rio Preto, Brazil

Corresponding author: Dr. Jose Maria Pereira de Godoy, E-mail: godoyjmp@gmail.com

\begin{abstract}
Lymphedema is a condition stemming from the accumulation of macromolecules in the interstitial space that leads to the retention of fluids. Patient seven-year old male patient with a diagnosis since birth of congenital primary lymphedema in the right lower leg underwent treatments over the years, but the condition progressed to elephantiasis. The patient sought the Godoy Clinic and was submitted to the intensive Godoy Method, nearly reaching normalization. Bioimpedance analysis was performed at the time and continued as a routine monitoring practice over the years, revealing a progression to subclinical systemic lymphedema. The increase in weight and the body mass index (BMI) over the years was accompanied by an increase in water, leading to the accumulation of total body water. An increase in weight in patients with lymphedema can evolve to subclinical systemic lymphedema, further aggravating the primary lymphedema.
\end{abstract}

Key words: Lymphedema; Subclinical; Elephantiasis; Treatment; Evolution

\section{INTRODUCTION}

Lymphedema is a condition stemming from the accumulation of macromolecules in the interstitial space that leads to the retention of fluids. This condition is divided into three clinical stages. In stage I, the patient awakens without edema, but swelling develops throughout the day. In stage II, the patient awakens with edema, which worsens throughout the day. Stage III is the aggravation of stage II and is also known as elephantiasis [1,2].

Godoy \& Godoy developed a new concept in the treatment of lymphedema that enables normalization or near normalization [2]. However, different factors can contribute to the aggravation of lymphedema, such as erysipelas, trauma and radiotherapy. Obesity has also been associated with lower limb lymphedema and is therefore yet another aggravating factor [3].
Animal studies have demonstrated that the increase in weight in obesity can trigger changes in the lymphatic pumping mechanism, capillary permeability and the immune system [3,4]. Bioimpedance analysis enables the determination of total intracellular and extracellular water as well as water in isolated extremities and the trunk and has demonstrated an increase in total body water with the progression of lymphedema [5].

This paper reports the evolution of bioimpedance findings with the increase in weight in a patient throughout a five-year period.

\section{CASE REPORT}

A 27-seven-year old male patient with a diagnosis since birth of congenital primary lymphedema in the right lower leg underwent treatments over the years,

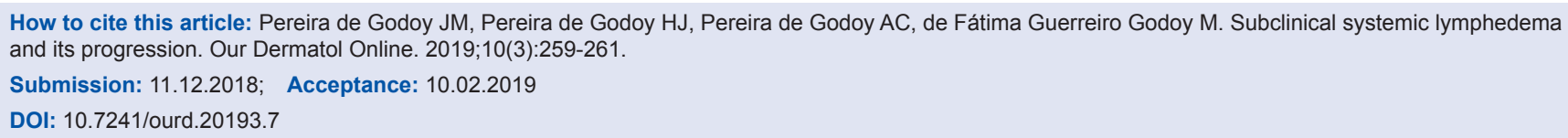


but the condition progressed to elephantiasis. The patient sought the Godoy Clinic and was submitted to the intensive Godoy Method, nearly reaching normalization (Figs. la and lb). Bioimpedance analysis was performed at the time and continued as a routine monitoring practice over the years, revealing a progression to subclinical systemic lymphedema. The increase in weight and the body mass index (BMI) over the years was accompanied by an increase in water, leading to the accumulation of total body water. However, the extracellular water/total body water ratio remained within the limits of normality, except in the leg with lymphedema (Table I).

\section{DISCUSSION}

The present study shows the progression in the accumulation of water in the entire body with the increase in BMI in a patient who had elephantiasis treated six years ago and remained in clinical stage II of lymphedema. With the increase in weight, an increase occurred in the amount of fluids accumulated in all limbs and the trunk, characterizing what we denominate subclinical systemic lymphedema [6]. At present, bioimpedance analysis has only detected lymphedema in the right leg, which corresponds to clinical stage II.

This study demonstrates that obesity is an aggravating factor of generalized edema, which was congenital primary lymphedema of the right lower limb in the present case. In clinical practice, we noticed that the increase in weight led to the emergence of lymphedema in the lower limb (determined by bioimpedance analysis) stemming from gravitational pressure, which progressed first to the trunk and then to the upper limbs.

In animal studies, an increase in BMI causes damage to the lymphatic pumping mechanism, changes in capillary permeability, an inflammatory process and changes in the immune defense system. These findings are compatible with those seen in humans with the increase in weight. A case study reports an association between lower limb lymphedema and generalized lymphedema.

The present study shows that these changes are systemic rather than localized, with generalized edema accompanying the increase in weight. Therefore, this study makes a novel contribution to the treatment of obesity and lymphedema.

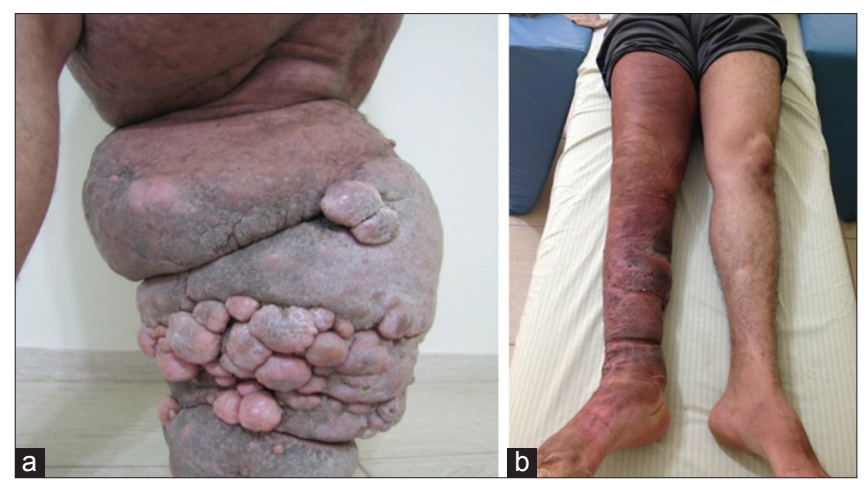

Figure 1: $(a$ and $b)$ Initial treatment and after with nearly normalization.

Table I: Evolution of total intracellular and extracellular water, water in limbs and trunk, and extracellular water (ECW)/total body water (TBW) ratio over five-year period

\begin{tabular}{lcccccc}
\hline Age & 27.0 & 28.0 & 29.0 & 30.0 & 31.0 & 31.0 \\
Height (kg) & 93.9 & 98.4 & 101.9 & 103.4 & 106.8 & 110.8 \\
Intracellular-water & 27.1 & 28.5 & 29.0 & 30.7 & 30.4 & 32.1 \\
Intracellular-Normal & 29.0 & 29.0 & 29.0 & 29.0 & 29.0 & 29.0 \\
Extracellular water & 17.3 & 18.2 & 18.2 & 18.9 & 18.0 & 19.9 \\
Extracellular-Normal & 17.8 & 17.8 & 17.8 & 17.8 & 17.8 & 17.8 \\
BMI & 30.3 & 31.8 & 32.9 & 33.4 & 34.5 & 35.8 \\
Water-R-arm & 2.49 & 2.60 & 2.65 & 2.81 & 2.80 & 2.95 \\
Water-L-arm & 2.39 & 2.59 & 2.61 & 2.77 & 2.78 & 2.90 \\
Water-Arm-Normal & 2.79 & 2.79 & 2.79 & 2.79 & 2.79 & 2.79 \\
Water-R-Leg & 9.54 & 9.17 & 10.19 & 10.32 & 10.41 & 11.09 \\
Water-L-Leg & 7.86 & 8.36 & 8.62 & 8.59 & 8.48 & 8.89 \\
Water-Leg-Normal & 7.77 & 7.77 & 7.77 & 7.77 & 7.77 & 7.77 \\
ECW/TBW-total & 0.390 & 0.390 & 0.387 & 0.381 & 0.382 & 0.384 \\
ECW/TBW-Normal & 0.390 & 0.390 & 0.390 & 0.390 & 0.390 & 0.390 \\
ECW/TBW-R-Arm & 0.370 & 0.374 & 0.365 & 0.367 & 0.367 & 0.365 \\
ECW/TBW-L- total & 0.372 & 0.375 & 0.365 & 0.367 & 0.367 & 0.365 \\
ECW/TBW-A-Normal & 0.39 & 0.390 & 0.390 & 0.390 & 0.390 & 0.390 \\
ECW/TBW-Trunk & 0.387 & 0.388 & 0.384 & 0.378 & 0.379 & 0.380 \\
ECW/TBW-Trunk-Normal & 0.39 & 0.390 & 0.390 & 0.390 & 0.390 & 0.390 \\
ECW/TBW-R-Leg & 0.416 & 0.405 & 0.410 & 0.405 & 0.408 & 0.414 \\
ECW/TBW-L-Leg & 0.377 & 0.390 & 0.377 & 0.367 & 0.366 & 0.366 \\
ECW/TBW-leg-Normal & 0.39 & 0.390 & 0.390 & 0.390 & 0.390 & 0.390 \\
\hline
\end{tabular}

\section{CONCLUSION}

An increase in weight in patients with lymphedema can evolve to subclinical systemic lymphedema, further aggravating the primary lymphedema.

\section{Consent}

The examination of the patient was conducted according to the Declaration of Helsinki principles.

\section{REFERENCES}

1. Lee BB, Antignani PL, Baroncelli TA, Boccardo FM, Ibrorson H, Campisi C, et al. Iua-Isvi consensus for diagnosis guideline of chronic lymphedema of the limbs. Int Angiol. 2015;34:311-32.

2. Pereira De Godoy JM, Amador Franco Brigidio P, Buzato E, 
www.odermatol.com

Fátima Guerreiro De Godoy M. Intensive outpatient treatment of elephantiasis. Int Angiol. 2012;31:494-8.

3. GD García Nores, DA Cuzzone, NJ Albano, GE Hespe, RP Kataru, JS Torrisi, et al. Obesity but not high-fat diet impairs lymphatic function. International J Obes. 2016;:1582-90.

4. Hespe GE, Kataru RP, Savetsky IL, García Nores GD, Torrisi JS, Nitti MD, et al. Exercise training improves obesity-related lymphatic dysfunction. J Physiol. 2016;594:4267-82.

5. de Godoy JMP, Pereira de Godoy HJ, Gracino de Marqui T, Spessoto LC, Godoy MFG. Mobilization of Fluids in the Intensive Treatment of Primary and Secondary Lymphedemas. Scien World J. 2018;2018:6537253.
6. de Godoy JMP, Pereira de Godoy LM, Ramos RR, Pereira de Godoy AC, de Fatima Guerreiro Godoy M. Subclinical systemic lymphedema caused by increased BMI in patients with lower limb lymphedema. Int J Med Scien Innov Res. 2018;3:170-6.

Copyright by Jose Maria Pereira de Godoy, et al.. This is an open access article distributed under the terms of the Creative Commons Attribution License, which permits unrestricted use, distribution, and reproduction in any medium, provided the original author and source are credited.

Source of Support: Nil, Conflict of Interest: None declared. 